\title{
Deforming glassy polystyrene: Influence of pressure, thermal history, and deformation mode on yielding and hardening
}

Article

Accepted Version

Vorselaars, B., Lyulin, A. V. and Michels, M. A. J. (2009) Deforming glassy polystyrene: Influence of pressure, thermal history, and deformation mode on yielding and hardening. The Journal of Chemical Physics, 130 (7). 074905. ISSN 00219606 doi: https://doi.org/10.1063/1.3077859 Available at https://centaur.reading.ac.uk/1557/

It is advisable to refer to the publisher's version if you intend to cite from the work. See Guidance on citing.

To link to this article DOI: http://dx.doi.org/10.1063/1.3077859

Publisher: American Institute of Physics

All outputs in CentAUR are protected by Intellectual Property Rights law, including copyright law. Copyright and IPR is retained by the creators or other copyright holders. Terms and conditions for use of this material are defined in the End User Agreement.

www.reading.ac.uk/centaur 
Central Archive at the University of Reading

Reading's research outputs online 


\title{
Deforming glassy polystyrene: influence of pressure, thermal history and deformation mode on yielding and hardening
}

\author{
Bart Vorselaars,,${ }^{1,2, *}$ Alexey V. Lyulin,,$^{1,3, \dagger}$ and M. A. J. Michels ${ }^{1,3}$ \\ ${ }^{1}$ Group Polymer Physics and Eindhoven Polymer Laboratories, \\ Technische Universiteit Eindhoven, P.O. Box 513, 5600 MB Eindhoven, The Netherlands \\ ${ }^{2}$ Department of Mathematics, University of Reading, Reading RG6 6AX, UK \\ ${ }^{3}$ Dutch Polymer Institute, P.O. Box 902, 5600 AX Eindhoven, The Netherlands
}

\begin{abstract}
The toughness of a polymer glass is determined by the interplay of yielding, strain softening and strain hardening. Molecular-dynamics simulations of a typical polymer glass, atactic polystyrene, under the influence of active deformation have been carried out to enlighten these processes. It is observed that the dominant interaction for the yield peak is of interchain nature and for the strain hardening of intrachain nature. A connection is made with the microscopic cage-to-cage motion. It is found that the deformation does not lead to complete erasure of the thermal history, but that differences persist at large length scales. Also we find that the strain-hardening modulus increases with increasing external pressure. This new observation cannot be explained by current theories such as the one based on the entanglement picture and the inclusion of this effect will lead to an improvement in constitutive modeling.
\end{abstract}

\section{INTRODUCTION}

Glassy polymeric materials show a rich behavior under deformation. During a compression test an initial elastic regime is followed by yield after which the material softens (strain softening, with a noticeable drop in stress). Upon compressing even further the strainhardening regime is entered; then the stress needed to deform the material grows. This is quantified by the strainhardening modulus, defined as the slope of the stress as function of Gaussian strain [1].

The relative magnitudes of these regimes have a dramatic effect on the resulting mechanical behavior of the polymer during a tensile test. For example, if the yield drop is high and the strain hardening is low, the material will be very brittle during uniaxial-stress extension. This is the case for the well-known atactic polystyrene (PS) glass. Here stress localization plays a role. If the weakest link of the material is strained at the start of the yield drop, the stress necessary to strain that part further will be lower than the stress to yield other parts of the material. Therefore, the weakest link will be strained further. If the strain hardening is insufficient to strengthen the weakest link this will ultimately break. As in this case the strain is limited to only a small part of the material the macroscopic sample shows an almost instantaneous fracture (i.e., within a few percent of extension). A minor change in the mechanical characteristics, i.e., a slight decrease in yield drop or more strain hardening, can result in a totally different behavior. Then a material could be easily be extended by one order of magnitude more before breakage.

The toughness is a variable which depends both on

\footnotetext{
*Electronic address: B.Vorselaars@reading.ac.uk

${ }^{\dagger}$ Electronic address: A.V.Lyulin@tue.nl
}

the polymer structure and on the combined thermal and mechanical history of the polymer material. Glassy polymers such as polyvinylchloride, polycarbonate (PC) and poly(methyl methacrylate) $[1,2]$ are more tough than PS, because they have a higher strain-hardening modulus. Brittle PS itself can also be made tougher, as was shown recently by Govaert et al.[3]. Such toughening can be reached by mechanical preconditioning or by thermal quenching of a polymer glass, thereby effectively decreasing the yield drop.

The thermal and mechanical history is therefore of prime importance in predicting the mechanical behavior of the polymer glass. Unfortunately a satisfying theory about the stress drop and the strain hardening of polymeric glasses is lacking. It is unknown what the exact reason is for the high yield tooth (peak and subsequent drop of the stress) observed in polystyrene. The other serious knowledge gap is the physical origin of strain hardening. Rubber-elasticity theory, based on the entropic picture of a polymer chain, predicts a strain-hardening modulus two orders of magnitude lower than what is measured experimentally $[4,5]$.

A better understanding of these two phenomena can be reached by studying microscopic properties. Despite the vast literature of experimental results on the mechanical properties of polystyrene, studies at the molecular level are rather scarce, as it is experimentally very hard to measure changes at this level. A successful alternative to study the mechanical properties of glassy materials is the method of molecular-dynamics simulations - physical details of all atoms are available and various parameters can be changed rather easily. For some mechanical properties such as the Young modulus, the yield peak and the strain-hardening modulus this has been successful, as being illustrated by numerous studies on various polymer models, such as on bead-spring models [6-13] and on bead-spring with bond-angle-potential models [14-17]. More chemically realistic MD simulations of polymers have been carried out on amorphous polyethylene (PE) 
[18-23], PS [24-27] and PC [24, 26-28]. Also other simulation techniques are applied to study the deformation of polymers, such as Monte Carlo algorithms or variants of energy-minimization methods for PE-alike[29], polypropylene [30, 31], poly(oxypropylene) [32], PC [33-35] and $\mathrm{PE}[36,37]$. As the simulation studies are limited to only small time and length scales, numerical agreement is often only possible by means of extrapolation over orders of magnitude.

Simulation studies on the phenomena we are interested in are, unfortunately, very limited. The importance of mechanical history is recently observed [27]. Simulations on chemically detailed [25-27] and model polymers $[16,17]$ showed that strain hardening can even occur for chains below the entanglement length contributing once more to the need for a view different from the rubberelasticity theory of the physical mechanism behind strain hardening.

In particular, the microscopic knowledge is still far from complete and the two questions raised above about the physical origins of the yield tooth and strain hardening are still open. The goal of the present study is to enhance the physical insight in these two phenomena. This is achieved by carrying out molecular-dynamics simulations on a chemically realistic atactic-PS melt. A chemically realistic model allows one to compare some simulation results with experiments and to do testable predictions. We thereby vary the external conditions: extension vs. compression, quenched vs. annealed, and as a function of external pressure. The reason for this variation is twofold. Firstly, it allows for extrapolations - these are necessary because computer power is only limited to small time and length scales. Secondly, we can study new parameter regimes - the variation of strain hardening with pressure is not known yet.

The remaining structure of this article is as follows. Simulation details will be discussed in §II. Afterwards the results of the different simulation conditions will be given. Subsequently they will be analyzed by means of energy and stress partitioning to see which interactions are dominant for the yield and the strain-hardening regime. Also the underlying forced microscopic cageto-cage motion will be studied. The consistent picture arising from these results is given in the summary and conclusions section, §VI. Ultimately these results should pave the way for new theories.

\section{SIMULATION DETAILS}

Molecular-dynamics simulations are carried out. Atactic polystyrene is simulated by using the force-field and sample preparation method as given in ref. 38 . In this way five independent samples are prepared at $T=540 \mathrm{~K}$. Temperature is controlled by using the socalled collisional-dynamics method ${ }^{39}$, in which the particles collide with 'virtual' particles of mass $m_{0}=0.1 \mathrm{Da}$ and with times between collisions described by a Pois- son process with average frequency $\lambda_{\text {coll }}=20 \mathrm{ps}^{-1}$. The density of the sample is set to $\rho=0.916 \mathrm{~g} \mathrm{~cm}^{-3}$, which equals the linearly extrapolated experimental density of PS at atmospheric pressure from lower temperatures ${ }^{40}$. After an equilibration of several ns, the internal pressure at this density is measured and is found to be equal to $42 \mathrm{MPa}$. Unless stated otherwise, simulations are carried out at this pressure. As the experimentally observed bulk compression modulus of PS is about $3 \mathrm{GPa}$ at room temperature $^{41}$, the pressure offset of $42 \mathrm{MPa}$ roughly corresponds to a small volume decrease of $1.4 \%$. A possible reason for this deviation from atmospheric pressure could be deviations in the force field; an example is that the excluded-volume potentials have a finite cut off. Nevertheless, we will also study the influence of external pressure on the mechanical properties. We will see further on that such an offset does not lead to a qualitatively different behavior. The pressure is maintained by using the Berendsen barostat ${ }^{42}$ for each side of the orthorhombic box independently, with a ratio of the time constant to the compressibility $\tau_{P} / \beta=0.011 \mathrm{Pas}$. The samples are then cooled to $T=300 \mathrm{~K}$ by $0.01 \mathrm{~K} \mathrm{ps}^{-1}$. The resulting density is $\rho=1.006 \mathrm{~g} \mathrm{~cm}^{-3}$. As an additional check for equilibration the characteristic ratio $C_{\infty}$ for PS is calculated and found to be 8.2 (with bondlength $l=1.53 \AA$, details to be published elsewhere), somewhat lower but close to $C_{\infty}=8.7-9.6$ resulting from smallangle neutron scattering experiments ${ }^{43}$ from a PS melt at $T=390-520 \mathrm{~K}$, and $C_{\infty}=9.9-10.2$ acquired by viscosity measurements around $308 \mathrm{~K}$ for various solvents ${ }^{44}$. To study the effect of cooling rate PS samples are also cooled to $T=300 \mathrm{~K}$ by $0.1 \mathrm{~K} \mathrm{ps}^{-1}$. We will call these the (computationally) quenched samples or (computationally) 'fast' cooled samples. This in contrast to the $0.01 \mathrm{~K} \mathrm{ps}^{-1}$ cooled samples, which we will call (computationally) slowly cooled, or (computationally) annealed samples. Usual experimental cooling rates are much slower. However, characteristics associated with processes such as activated ones typically vary linear with the logarithm of the cooling rate and therefore the difference with experiments as well. If not specified, results are for the computationally slowly cooled samples.

The mechanical tests at $T=300 \mathrm{~K}$ (about room temperature) are carried out in the following way. We consider both uniaxial-stress extension and uniaxial-stress compressions, implemented by resizing the size of the periodic orthorhombic box in the axial direction in a prescribed manner and by keeping the lateral sizes at a constant stress value by using the Berendsen barostat. The initial box sizes are around $L(0)=50 \AA$. The coordinate system is oriented by taking the $x$-axis along the active extension or compression direction. The engineering strain is then $\varepsilon_{\text {eng }}=\frac{L_{x}(t)-L_{x}(0)}{L_{x}(0)}$. Other symbols which will be used throughout this article are the true strain, $\varepsilon_{\text {true }}=\ln \left(1+\varepsilon_{\text {eng }}\right)$ and the draw ratio, $\lambda=1+\varepsilon_{\text {eng }}$. For small strains $\varepsilon_{\text {eng }} \approx \varepsilon_{\text {true }}$ and in this case the strain is simply written as $\varepsilon$. All uniaxial-stress extension simulations are at a constant velocity of $0.01 \AA \mathrm{ps}^{-1}$, corre- 
sponding to a deformation rate of $\dot{\varepsilon}_{\text {eng }} \approx 2 \times 10^{8} \mathrm{~s}^{-1}$ (here the dot means differentiation with respect to time). The compression simulations are at a constant deformation rate of $\dot{\varepsilon}_{\text {true }}=-10^{8} \mathrm{~s}^{-1}$. Next to constant velocity, some extension simulations are also realized at a constant deformation rate with $\dot{\varepsilon}_{\text {true }}=10^{8} \mathrm{~s}^{-1}$. A direct comparison with the constant-velocity extension simulations shows that the difference in the resulting stress-strain relation is below the statistical fluctuations and therefore we will only present the extension results with constant velocity. To increase statistics the deformation is carried out three times for each sample (along each axis once), making the total number of runs equal to 15 for each set of external conditions (if unspecified). Some simulations are realized at pressures different from the offset value. This is established by taking the slowly cooled samples at $300 \mathrm{~K}$ and setting the external pressure to the desired value. This is followed by an equilibration of $0.5 \mathrm{~ns}$. These samples then either serve as an input for the deformation run or for the unstrained production run.

In the current study each sample consists of 8 PS chains of 80 monomer units each. This is below the entanglement length $N_{e}$ of PS (simulations ${ }^{45}$ at $T=450 \mathrm{~K}$ : $N_{e}=83$ monomers; experiments ${ }^{46}$ at $T=413 \mathrm{~K}$ : $N_{e}=128$ monomers and ${ }^{47}$ at $T=490 \mathrm{~K}: N_{e}=139$ monomers, determined by measuring the rubbery-like plateau modulus $G_{N}$ of an entangled PS melt above the glass transition and using this value to calculate the molecular weight between entanglements $M_{e}$ by means of $^{48} M_{e}=\frac{4}{5} \rho R T / G_{N}$, with $R$ the universal gas constant). Usually deformation would be affine for large length scales; for very long chains the ends do not feel immediately the connectivity constraint of each other as they are separated by many segments. For short chains this is not the case, and deformation becomes more nonaffine; the end-to-end distance does not change in the same way as the box sizes. This could have an effect on the determination of the strain hardening. In our previous study ${ }^{26}$ we saw, however, that by doubling the chain length the strain hardening modulus does not change in the studied strain range (until about $100 \%$ extension) and is comparable to even longer chains for the typical strain rates in use. This is also confirmed by other simulation studies of a toy polymer model by Hoy et al. ${ }^{16}$. Their study showed that the change in the end-to-end distance for a (short) chain of length $N \approx N_{e}$ was more than $80 \%$ of the affine value (here $0 \%$ is taken to be if the chains do not change in size at all) at $\left|\lambda^{2}-\lambda^{-1}\right|=2.6$ $\left(\left|\varepsilon_{\text {true }}\right| \approx 1\right)$ under uniaxial compression, and that the resulting strain-hardening modulus for these short chains was nearly the same as for the much longer chains. In summary, we feel that the present short-chain simula- tions should give relevant insight into strain hardening in general.

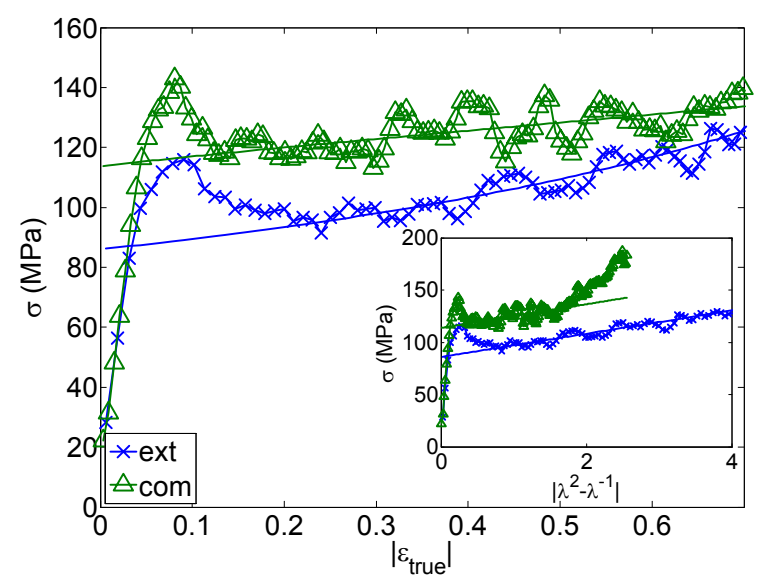

Figure 1: Von Mises true stress vs. absolute true strain for atactic polystyrene during extension ( $\dot{\varepsilon}_{\text {eng }}=2 \times 10^{8} \mathrm{~s}^{-1}$, 'ext') and compression ( $\dot{\varepsilon}_{\text {true }}=-10^{8} \mathrm{~s}^{-1}$, 'com'). Inset shows the von Mises stress as a function of $\left|\lambda^{2}-\lambda^{-1}\right|$, thereby showing a wider range for the compression data. Fitting the data in the inset by eq. 2 in the range $\left|\varepsilon_{\text {true }}\right|=0.2-0.6$ gives the value of the yield stress for extension $\sigma_{Y}=86 \mathrm{MPa}$ and strainhardening modulus $G_{h}=11 \mathrm{MPa}$, and for compression $\sigma_{Y}=$ $114 \mathrm{MPa}$ and $G_{h}=11 \mathrm{MPa}$.

\section{STRESS DEVELOPMENT DURING DEFORMATION}

In this section we will look at the stress development of polystyrene during deformation under various conditions. At first, we compare compression and extension simulations with each other and with the literature, in order to check if the simulations are resembling basic experimental results. The second condition to vary is the thermal history, as it is known experimentally that the yield tooth is highly influenced by this. The final external factor of interest is the pressure, which turns out to greatly affect the strain-hardening modulus.

\section{A. Compression and extension}

During uniaxial-stress extension and compression tests the stress tensor is monitored. Instead of looking at all components of the stress tensor it is customary to study a measure of the second invariant of the stress tensor, the von Mises equivalent true stress ${ }^{55}$

$$
\sigma_{\mathrm{vM}}=\sqrt{\frac{1}{2}\left(\sigma_{x}-\sigma_{y}\right)^{2}+\frac{1}{2}\left(\sigma_{y}-\sigma_{z}\right)^{2}+\frac{1}{2}\left(\sigma_{z}-\sigma_{x}\right)^{2}+3 \sigma_{x y}^{2}+3 \sigma_{y z}^{2}+3 \sigma_{z x}^{2}}
$$


Table I: Results from simulation and literature experiments of various mechanical properties of PS.

\begin{tabular}{|c|c|c|c|c|c|c|c|}
\hline property & eq. & this work & condition $^{a}$ & fig. & experiments & condition $^{b}$ & ref. \\
\hline \multirow[t]{3}{*}{$\sigma_{\text {peak }}(\mathrm{MPa})$} & & $143 \pm 4$ & compr. & 1 & 87,100 & compr., var. rates ${ }^{c}$ & 3,49 \\
\hline & & $117 \pm 4$ & ext., sc & 1 & 92 & compr., sc & 50 \\
\hline & & $104 \pm 4$ & ext., fc & 2 & 72 & compr., $\mathrm{fc}^{d}$ & 50 \\
\hline \multirow[t]{3}{*}{$\varepsilon_{\text {eng }}($ peak $)$} & & -0.08 & compr. & 1 & & & \\
\hline & & 0.09 & ext., sc & 1 & -0.057 & compr., sc & 50 \\
\hline & & 0.12 & ext., fc & 2 & -0.063 & compr., $\mathrm{fc}^{d}$ & 50 \\
\hline \multirow[t]{3}{*}{$\sigma_{Y}(\mathrm{MPa})$} & 2 & 114 & compr. & 1 & & & \\
\hline & & 86 & ext., sc & 1 & & & \\
\hline & & 86 & ext., fc & 2 & & & \\
\hline \multirow[t]{3}{*}{$G_{h}(\mathrm{MPa})$} & 2 & 11 & compr. & 1 & $9,11,13$ & compr., var. rates ${ }^{e}$ & $4,49,51$ \\
\hline & & 11 & ext., sc & 1 & & & \\
\hline & & 11 & ext., fc & 2 & & & \\
\hline$\mu_{Y}$ & 3 & 0.17 & & 4 & $0.14,0.22$ & & 49,52 \\
\hline$\sigma_{\mathrm{vM}, Y, P_{Y}=0}(\mathrm{MPa})$ & 3 & 86 & & 4 & & & \\
\hline$\mu_{h}$ & 4 & 0.045 & & $5(\mathrm{a})$ & & & \\
\hline$G_{h, P_{Y}=0}(\mathrm{MPa})$ & 4 & 8.6 & & $5(\mathrm{a})$ & & & \\
\hline$\nu$ & 7 & $0.35 \pm 0.02$ & & 8 & $0.33,0.37-0.38$ & & 53,54 \\
\hline
\end{tabular}

${ }^{a}$ Sc: relatively slowly cooled (for simulations $\dot{T}=-0.01 \mathrm{~K} \mathrm{ps}^{-1}$ ); fc: relatively fast cooled (for simulations $\dot{T}=-0.1 \mathrm{~K} \mathrm{ps}^{-1}$ )

${ }^{b}$ If not given, $\dot{\varepsilon}_{\text {true }}=-10^{-3} \mathrm{~s}^{-1}$

${ }^{c} \dot{\varepsilon}_{\text {true }}=-10^{-3}$ and $-10^{-2} \mathrm{~s}^{-1}$ respectively

${ }^{d}$ Cooling velocity in the order of $\mathrm{mK} \mathrm{s}^{-1}$

${ }^{e} \dot{\varepsilon}_{\text {true }}=-10^{-3},-10^{-3}$ and $-10^{-2} \mathrm{~s}^{-1}$ respectively

The measured von Mises stress as a function of the applied strain during a uniaxial-stress extension and a uniaxial-stress compression simulation is depicted in fig. 1. Results are very similar if the true stress in the axial direction is plotted instead of the von Mises true stress (not shown). Each marker in fig. 1 represents an average of the measured stress during a period of time corresponding to the separation between two subsequent markers. In addition to this, the stress is averaged over 15 runs. The standard deviation of the average stress near the yield peak is about $4 \mathrm{MPa}$ and increases slightly for larger strains (near $\left|\varepsilon_{\text {true }}\right|=0.7$ it is about $7 \mathrm{MPa}$ ).

Regarding the behavior near the yield point we observe that the stress both at the yield peak $\sigma_{\text {peak }}$ and near the yield drop after the peak are higher under compression than under tension (see fig. 1 and table I). The same trend in yield stress was observed in a molecularmechanics simulation of atactic poly(oxypropylene) ${ }^{32}$. The higher hydrostatic pressure under compression than under tension is likely the reason for a higher yield stress (as yield stress usually increases with increasing pressure $\left.^{56}\right)$.

Experimental yield peak values under compression at room temperature are slightly less (see $\sigma_{\text {peak }}$ in table I). The reason for the deviation is that the deformation conditions are not the same: the experiments have a much slower strain rate (thereby lowering the yield stress; experimentally it is observed that the yield stress has a logarithmic dependence on the strain rate ${ }^{49}$ ), slower cool- ing rate (resulting in a higher yield stress ${ }^{50}$ ), and longer chain lengths (increasing the yield stress due to the slower relaxation of the middle of the chain compared to the chain ends ${ }^{57}$ ). Despite these quantitative differences, the results show qualitatively the same behavior.

Let us now focus on the strain hardening modulus. We use a simple constitutive relation between the (true) stress $\sigma$ and the strain after yielding to determine it, the Gaussian-based equation ${ }^{58,59}$

$$
\sigma=\sigma_{Y}+G_{h}\left(\lambda^{2}-\lambda^{-1}\right)
$$

with $G_{h}$ is the strain-hardening modulus, and $\sigma_{Y}$ is the offset yield stress (which is lower than the yield peak value, $\left.\sigma_{\text {peak }}\right)$. We limit the fit range for extensions to $\left|\varepsilon_{\text {true }}\right|=0.25-0.6$ (as for $\varepsilon_{\text {true }}>0.6$ samples break) and to allow for a better comparison the fit range for compression is restricted to the same range in terms of the absolute value of the true engineering strain. The mechanical moduli $\sigma_{Y}$ and $G_{h}$ resulting from the fit are given in table $\mathrm{I}$.

In eq. 2 the stress is linear with the Gaussian strain $\lambda^{2}-\lambda^{-1}$, both for extension and for compression. To check this, the stress is also plotted as a function of the absolute value of the Gaussian strain, see inset in fig. 1 . The tension test shows indeed a linear regime after initial yield. The compression data deviate from this fit for large strains; extending the fit range to include all data points at large strains would result in a much larger apparent strain-hardening modulus, $G_{h}=37 \mathrm{MPa}$. We will come 


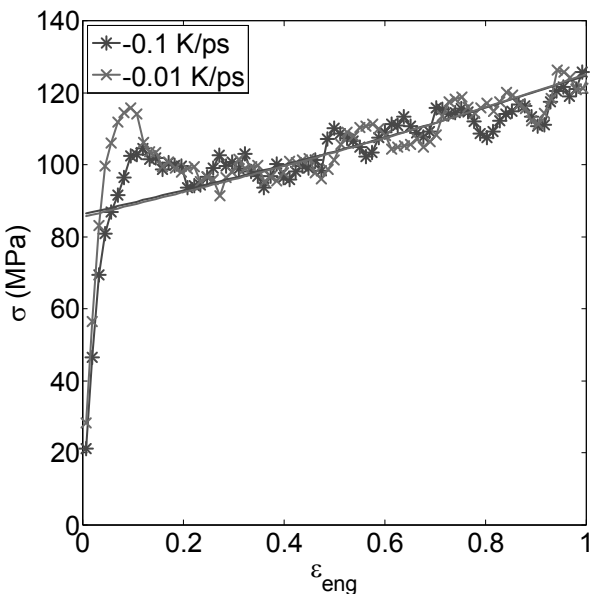

Figure 2: Von Mises equivalent true stress vs. strain for atactic polystyrene for two different cooling rates. Solid lines are fits of eq. 2. There is no difference for the strain-hardening modulus and the extrapolated yield value. However, the initial yield peak is higher for the slowly cooled sample. Also there is a small difference in the strain at which the yield peak occurs (numerical values are given in table I).

back to this point in $\S \mathrm{VC}$, as there the stress will be partitioned into smaller parts to isolate which interaction is responsible for this effect.

The experimental values for the strain-hardening modulus $G_{h}$ for room-temperature PS under compression are around the values as found by the present compression and extension simulations (table I). Good extension data are not available, as during an extension experiment PS samples usually break. We observe quantitative similarity between the simulation and the experimental strain hardening moduli; the agreement is much closer than for the yield stress values. A possible reason might be the following. Experimentally it is known that the strainhardening modulus of some polymeric materials increases with increasing strain rate; examples are high-density polyethylene $^{60}$, and polyurea ${ }^{61}$. However, for other polymers the increase is very small or nearly absent, such as for polycarbonate ${ }^{62}$. The same might be applicable for PS, so that both simulation and experiment render the same strain-hardening modulus.

In spite of the obvious differences in deformation conditions, we can conclude that the simulated polymer system shows the same qualitative behavior as polymers do in experimental studies. Hence the simulation model in use is realistic enough in terms of reproducing mechanical properties of polystyrene and we can proceed to analyze the results in more detail.

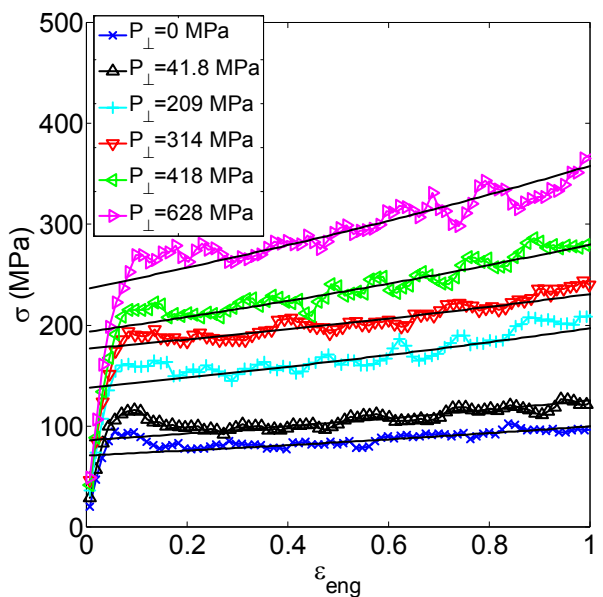

Figure 3: Von Mises stress vs. strain during uniaxial-stress extension for different external pressures at $T=300 \mathrm{~K}$. Solid lines are fits to the simulation data as is done in fig. 1. Observe that both the yield peak and the strain-hardening modulus turn out to increase with increasing external pressure.

\section{B. Quenched vs. annealed samples}

The properties of a glassy material are much affected by the thermal history of the sample, i.e., the initial thermal treatment before deformation starts. In particular the mechanical behavior is affected, see fig. 2. Two different scenarios are compared: samples cooled down by $0.1 \mathrm{~K} \mathrm{ps}^{-1}$ ('fast' cooled) and samples cooled down by $0.01 \mathrm{~K} \mathrm{ps}^{-1}$ ('slowly' cooled). For the fastercooled sample (more quenched) the yield peak is lower than for the slower-cooled sample ( $\sigma_{\text {peak }}$, table I). As discussed, this tendency is confirmed by experimental results on polystyrene under compression (again, table I). From the simulations the strain at the yield peak is around $9 \%$ for the slowly cooled sample, and $12 \%$ for the faster-cooled sample. Experimental results show a smaller strain value at yield $(6 \%$, ref. 50$)$. The trend is the same; also experimentally the quenched sample has a slightly higher strain at yield as compared to the annealed sample ${ }^{50}\left(\varepsilon_{\text {eng }}\right.$ (peak), table I). Within statistical error the strain-hardening modulus is not altered by a different thermal history, the strain-hardening modulus is $G_{h}=11 \mathrm{MPa}$ for both cooling scenarios. We conclude that the simulated aging effects are qualitatively similar to experiments.

\section{Influence of external pressure}

The behavior of $\sigma_{Y}$ as a function of external factors such as temperature $T$ and pressure $P$ is well known. The effects of these external factors are described by the Eyring equation ${ }^{56}$. Even so, much less understanding is available for $G_{h}$. If rubber theory ${ }^{59,63}$ would be valid for 
glassy polymers, $G_{h}$ equals $\frac{k_{B} T \rho}{M_{e}}$. Here $\rho$ is the mass density and $M_{e}$ the molecular weight between entanglements. Note that the predicted strain-hardening modulus does not depend explicitly on external pressure and it increases linearly with temperature. Experimentally the trend is, however, opposite; it is found that the strainhardening modulus is decreasing with temperature ${ }^{64}$.

A different way of reasoning is that the strainhardening modulus actually depends in the same way as the yield stress on external factors such as temperature and strain rate. Regarding the temperature dependence it indeed has been found experimentally that the ratio $\sigma_{Y} / G_{h}$ is fairly constant over a range of temperatures ${ }^{1,59}$. Simulations have shown ${ }^{15}$ that also the dependence on strain rate is about the same for both $\sigma_{Y}$ and $G_{h}$.

What about the influence of external pressure? As far as the authors are aware the effect of external pressure on the strain hardening has not been studied before, neither experimentally nor in simulations. It is known that the yield peak increases with increasing external pressure. Based on the observed communalities between the dependence of yielding and strain hardening on temperature and strain rate, one expects that the strain hardening modulus would also increase with external pressure.

Simulations have been carried out at various external pressures to test this. In fig. 3 the von Mises stress is plotted as a function of strain for six imposed lateral pressures (number of simulation runs per pressure point is at least 10). As with experimental data the simulation results show that the yield stress increases with external pressure. Note that the strain-hardening modulus also increases with increasing external pressure. As just stated, this is in contrast to what one would expect on the basis of the rubber theory ${ }^{59}$, in which the strain-hardening modulus $G_{h}=\frac{k_{B} T \rho}{M_{e}}$ does not explicitly depend on the external pressure. Even an implicit pressure dependence caused by a change in density cannot explain the change in the strain hardening modulus.

To make more quantitative statements we plotted the pressure dependence of the yield stress and the strain hardening modulus. The yield stress results are plotted in fig. 4, where the von Mises yield stress $\sigma_{\mathrm{vM}, Y}$ is shown.

The values of the yield stress $\sigma_{\mathrm{vM}, Y}$ are determined by fitting eq. 2 to the accompanying stress-strain curve. For the abscissa the pressure near the start of yielding, $P_{Y}=\frac{2}{3} P_{\perp}-\frac{1}{3} \sigma_{\|, Y}$, is used. Various other definitions of a yield stress exist ${ }^{56}$, but the currently used one is chosen as it is not so susceptible to noise.

To determine the proportionality between the yield stress and pressure we fit the data in fig. 4 by the common relation

$$
\sigma_{\mathrm{vM}, Y}\left(P_{Y}\right)=\sigma_{\mathrm{vM}, Y, P_{Y}=0}+\sqrt{3} \mu_{Y} P_{Y} .
$$

Here $\sigma_{\mathrm{vM}, Y, P_{Y}=0}$ and $\mu_{Y}$ are fit coefficients (the same convention for $\mu_{Y}$ has been adopted as by, e.g., ref. 49 , hence the factor $\sqrt{3}$ ). The constant $\mu_{Y}$ is known as the pressure coefficient for yielding a material and can be interpreted as some kind of internal friction

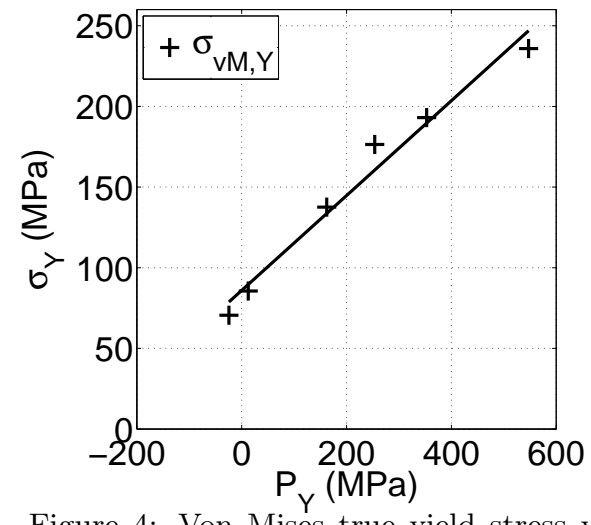

Figure 4: Von Mises true yield stress vs. pressure near the yield point. Solid line is a fit to eq. 3 .

coefficient ${ }^{10}$, in analogy with the proportionality constant in the Amontons-Coulomb law, which relates the friction force to the normal force for sliding two materials over each other under the influence of this normal force $^{55}$. The results from a least-squares fit of the data with eq. 3 are given in table I $\left(\mu_{Y}\right.$ and $\left.\sigma_{\mathrm{vM}, Y, P_{Y}=0}\right)$. Although the value of $\mu_{Y}$ depends on the exact method of extracting a yield stress ${ }^{10}$, the experimental values of atactic PS are close to the simulation results (table I).

For the strain hardening dependence we will also use a linear relation for describing $G_{h}\left(P_{Y}\right)$, similar to $\sigma_{\mathrm{vM}, Y}\left(P_{Y}\right)($ eq. 3$)$

$$
G_{h}\left(P_{Y}\right)=G_{h, P_{Y}=0}+\mu_{h} P_{Y}
$$

with $G_{h, P_{Y}=0}$ the strain-hardening modulus for the case that the pressure at yield is equal to zero and $\mu_{h}$ the pressure-dependency factor of the strain-hardening modulus. We find $\mu_{h}=0.045$ and $G_{h, P_{Y}=0}=8.6 \mathrm{MPa}$ (see also table I).

In literature it has been proposed that $\sigma_{Y}$ and $G_{h}$ are coupled, i.e., eq. 2 is written as ${ }^{15}$

$$
\sigma=\sigma_{Y}(T, P, \dot{\varepsilon}) F(\lambda)
$$

in which only $\sigma_{Y}$ is influenced by temperature, pressure and deformation rate, while $F(\lambda)=\left(1+\frac{G_{h}}{\sigma_{Y}}\left(\lambda^{2}-\lambda^{-1}\right)\right)$ and thus $\frac{G_{h}}{\sigma_{Y}}$ depends on other intrinsic polymer-specific properties. Our simulation results with various values of the external pressure do not exclude this multiplicative form of the stress-strain relation, see fig. 5(b). In fact, it favors this type of stress-strain-relation over the type in which the strain-hardening modulus is independent of the external pressure. 


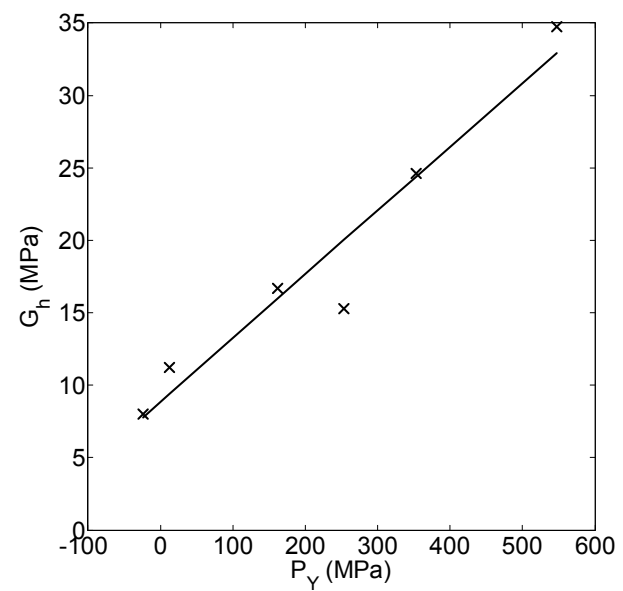

(a)

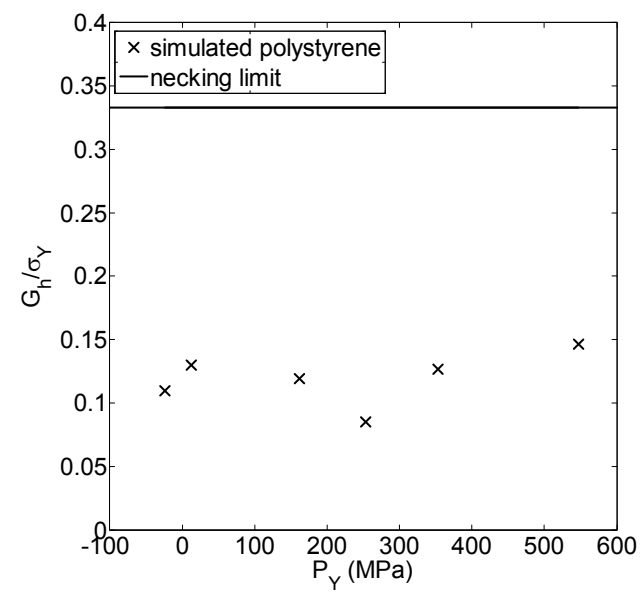

(b)

Figure 5: (a) Strain-hardening modulus vs. pressure near yield. (b) The ratio of strain-hardening modulus to von Mises true yield stress vs. yield pressure. Considère's limit for necking, $G_{h} / \sigma_{Y}=1 / 3$, is also shown.

One could think that the material becomes tougher upon an increase in the strain-hardening modulus. According to Considère's construction it is the ratio $G_{h} / \sigma_{Y}$ which determines the toughness of the material ${ }^{1}$, not $G_{h}$ alone. The Considère limit for necking, $G_{h} / \sigma_{Y}=\frac{1}{3}$, is also plotted in fig. 5(b). We observe that despite the increase in $G_{h}$, the polymer will not become much tougher upon applying an external pressure.

\section{ENERGETICS}

\section{A. Work and dissipation}

More insight into yielding and hardening can be acquired by looking at the energetics during the deforma-

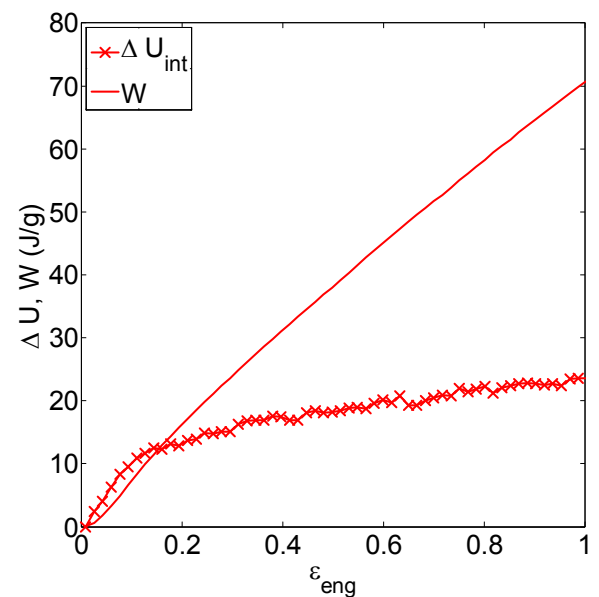

(a)

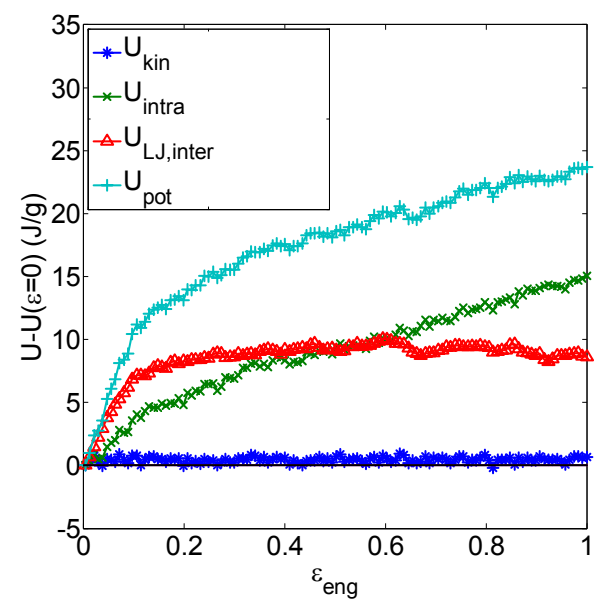

(b)

Figure 6: (a) Applied work $W$ and increase in internal energy $\Delta U_{\text {int }}$ vs. strain. (b) Various contributions to the internal energy as a function of strain during uniaxial-stress extension.

tion process. A part of the work needed to extend a sample is stored in the material (elastic response) and another part will be dissipated (viscous response). In what way is this energy stored, and how much of the energy is dissipated? To answer these questions we first look at the evolution of the work and total internal energy and afterwards at the further partitioning of this internal energy into smaller components.

The amount of work $W$ done on the sample is determined by calculating the product of the net force on a side of the orthorhombic box and the displacement of that side for all three perpendicular directions of the box during deformation ${ }^{65}$, as the off-diagonal elements of the strain tensor are approximately zero for our deformation 
$\operatorname{mode}{ }^{66}$

$$
\begin{aligned}
d W & =F_{x} \mathrm{~d} L_{x}+F_{y} \mathrm{~d} L_{y}+F_{z} \mathrm{~d} L_{z} \\
& =\sigma_{x} L_{y} L_{z} \mathrm{~d} L_{x}+\sigma_{y} L_{z} L_{x} \mathrm{~d} L_{y}+\sigma_{z} L_{x} L_{y} \mathrm{~d} L_{z} \\
& =V\left(\sigma_{x} \mathrm{~d} \varepsilon_{x}+\sigma_{y} \mathrm{~d} \varepsilon_{y}+\sigma_{z} \mathrm{~d} \varepsilon_{z}\right)
\end{aligned}
$$

where $\varepsilon_{x}$ equals $\frac{1}{L_{x}} d L_{x}, \sigma_{x}$ the true-stress component along the $x$-axis, and similar for the $y$ and $z$ components. The total work done on the sample during deformation is the integral $W=\int_{0}^{\varepsilon} d W$ where the integration has to be carried out from the initial undeformed state to the final deformed state.

This work $W$ and the increase of the internal energy $\Delta U_{\text {int }}$ as a function of engineering strain $\varepsilon_{\text {eng }}$ is shown in fig. 6(a). Up to about $15 \%$ strain all work done on the sample is converted into internal energy. The internal energy even rises faster than the amount of work done on the sample. This is a well-known effect under small extensions ${ }^{59,67}$. Under these small extensions the temperature of the material usually drops: the JouleThomson effect. But as our sample is immersed in a heat bath (the thermostat), there is a net heat flow into the sample, nullifying the temperature drop.

For larger strains the internal energy keeps increasing, but most of the work is now converted into heat. This means that during the flow of the material almost all energy is dissipated. Only a fraction of the work is converted into internal energy. This is in accordance with experimental results for PS; the percentage of work which is converted into internal energy decreases after the initial yielding ${ }^{68}$. In an MD simulation study of a toy-model polymer $^{16}$ the dissipative stress was monitored and a similar conclusion was reached.

\section{B. Internal-energy partitioning}

To see where this extra internal energy is stored, the components of it are monitored during deformation, see fig. 6(b). The internal energy $U_{\text {int }}$ is composed of a kinetic part $U_{\text {kin }}$ and a potential part $U_{\text {pot }}$. The potential energy is further partitioned into interchain energy $U_{\text {LJ,inter }}$ and intrachain energy $U_{\text {intra }}$. The interchain energy consists only of Lennard-Jones (LJ) interactions and the intrachain energy is made of intrachain LJ, 2-particle covalent-bond (12), 3-particle covalent-angle (13) and 4particle torsion (14) interactions. Due to the complexity of the side groups and phenyl-phenyl interactions in PS further partitioning into separate 12, 13, 14 and intra-LJ interactions does not lead to much more understanding about glassy polymers in general and therefore we will not show these results here. Interactions of chains with its images can be neglected in our simulations, because the sum of the length of the LJ interaction (about the LJ cut-off distance) and the extent of a chain (the mean square end-to-end distance) is less than the box size. Up to about $10 \%$ extension the main increase is in the interchain Lennard-Jones interactions. After initial yielding only the intrachain energy contribution continues to rise, while the interchain contributions saturate to a value of almost $10 \mathrm{~J} \mathrm{~g}^{-1}$. The kinetic-energy term stays approximately constant (due to the thermostat).

Possible causes of the increase in intrachain energy could be the following. Upon extending the sample the chains become more extended as well. The covalent bonds (the stiffest springs in the system) will initially give rise to the highest energy increase. However, as they impose an opposite force they will drag other particles to relax the stress. Then other mechanisms for making the chain more extended will become active (such as bending the valence angles and changing conformations from gauche to trans states). Nevertheless, the opposite force of the covalent bond will increase the energetic contribution of that interaction. So is the case for other interactions, such as for bond-angle bending interactions and torsion interactions. Also the energy from the intrachain LJ interactions is likely to increase, for the following reason. In a trans configuration the two phenyl rings of a meso dyad repel each other because of their close distance; they feel a strong repulsive LJ interaction. An extended chain has more trans configurations and therefore this could lead to an increase in the intrachain LJ energy.

The reason for the initial increase in interchain LJ energy and saturation afterwards can be understood from the breaking of LJ bonds upon flowing. Here a pair of particles is considered to be bonded by a LJ interaction (called a LJ bond) if the separation between them is small enough, so that the energy necessary to separate the two particles from each other is a non-negligible fraction (say about $10 \%$ ) of the well depth of the LJ interaction. The breaking of LJ bonds upon flowing will be treated later on in more detail when the samples of different thermal history will be discussed.

Similar trends in energy contributions have been found in other simulation studies. Also in a Monte Carlo simulation of a uniaxially compressed network ${ }^{29}$ the intermolecular potential energy increases a lot up to yield and hardly increases after yield. Furthermore, the intramolecular energies continue to increase as well after yield. This suggests that the observed behavior is rather general. In molecular-mechanics simulations of atactic poly(oxypropylene) ${ }^{32}$ the dominant change in the energy near the initial yield point was likewise ascribed to the van der Waals energy, although no distinction was made between intrachain and interchain energy.

\section{Influence of thermal history}

In contrast to the observed influence of the thermal history on the yield peak (fig. 2), the strain-hardening modulus does not change with cooling rate: both cooling scenarios gave the same value of $G_{h}=11 \mathrm{MPa}$. From the identical behavior of the two thermally different materials after yield it was proposed that the aging history is erased 


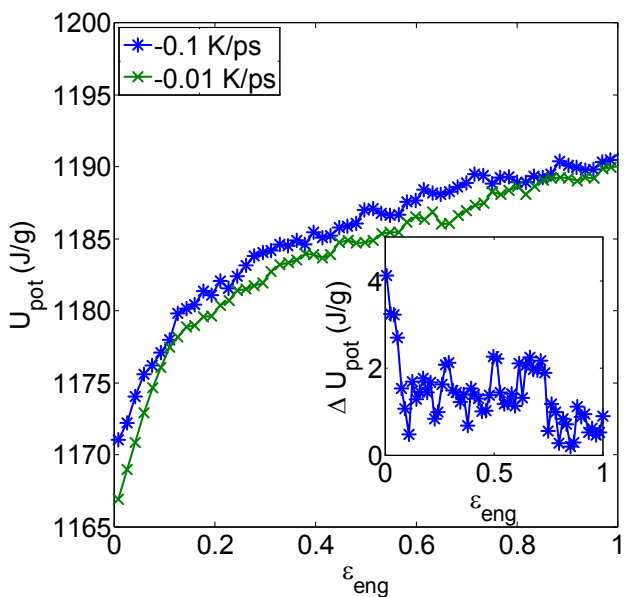

(a)

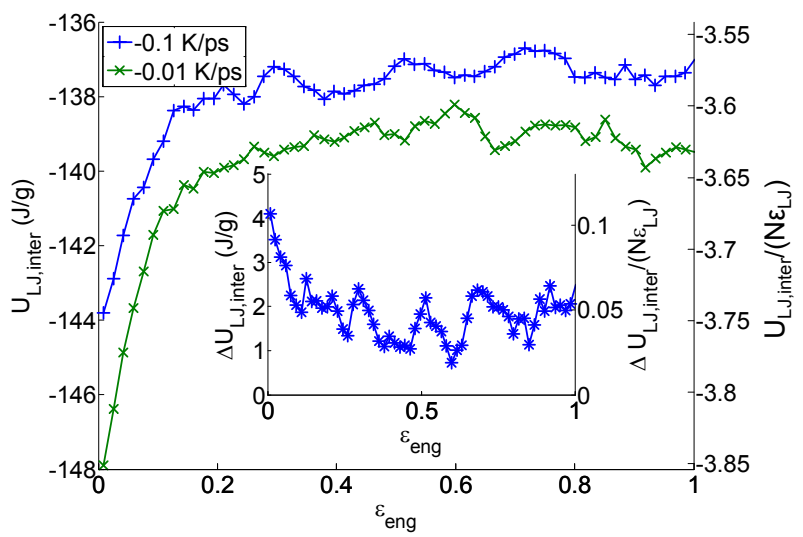

(b)

Figure 7: Plotted is an energy vs. engineering strain for atactic PS produced with two different cooling rates. Insets shows the difference. (a) Total potential energy. The initial difference in potential energy does not vanish entirely upon deformation. The main decrease occurs up till shortly after the yield point $\left(\varepsilon_{\text {eng }} \approx 0.1\right)$. In the strain-hardening regime the difference stays approximately the same. (b) Total interchain energy (both in units of $\mathrm{Jg}^{-1}$ and $\epsilon_{L J}$, see text). The interchain energy behaves very similar to the potential energy.

completely from this point ${ }^{59}$ and that deformed annealed samples are behaving similar as quenched samples (hence the term mechanically-induced rejuvenation). However, only one property has been compared, the stress. Are other properties of the material also equivalent after initial yielding? To answer this question we will isolate the interaction type that is responsible for the difference in yield peak.

We will first compare the potential energy during deformation for samples with different cooling history. As was shown, the potential energy $U_{\text {pot }}$ plays a large role for initial yield. The difference between the fast and the slower-cooled sample in terms of this energy is depicted in fig. $7(\mathrm{a})$. Prior to deformation $U_{\text {pot }}$ is lower for the slower-cooled sample, as is typical for glassy materials ${ }^{69}$, which are in a non-equilibrium state. The slower-cooled sample has more time to equilibrate and falls out of equilibrium at a lower temperature. It is therefore closer to the equilibrium state, of lower energy. The slower-cooled sample has more time to find deeper minima of the energy landscape. The decrease in energy towards the equilibrium value is sometimes also interpreted as an increase in local ordering, as then the difference from the underlying crystalline structure (if any) is smaller (in terms of the total energy).

During deformation the difference in potential energy between the two samples of varying cooling scenario changes. For strains up to about $10 \%$ the potentialenergy difference decreases and for larger strains the difference saturates to an approximately constant value. Note that the energy difference does not vanish entirely, illustrating that the deformation of a sample does not completely erase the aging history. A similar observation has been made before for smaller strain values ${ }^{27}$. Despite this incomplete erasure, the strain-hardening modulus is apparently not affected (fig. 2).

What would be the main difference in potential energy between the two cooling scenarios? In a previous study of atactic $\mathrm{PS}^{27}$ it was seen that the difference between quenched and annealed samples was evenly distributed among the various interactions (bond stretching, bond bending, torsion and total LJ energy). The present study goes beyond that work, by partitioning the total LJ energy even further into an intrachain and an interchain part, so that we can discriminate between inter- and intrachain effects. This is important, as the total LJ energy is heavily influenced by the local intrachain chemistry. We observe that the interchain LJ interaction is the dominant one, recognizable by comparing the insets in fig. 7(b) and fig. 7(a). Although the fluctuations are quite high $\left( \pm 1.6 \mathrm{~J} \mathrm{~g}^{-1}\right.$ and $\pm 2.0 \mathrm{~J} \mathrm{~g}^{-1}$ for the slowly and faster cooled samples) we still think this observation is significant. It is also expected that the interchain LJ energy is the major contribution, because the slowly and fastercooled samples differ mainly in the initial yield region, and the main contribution to the increase in potential energy near initial yield is due to interchain interactions. The observation that the total LJ interaction is not dominating is due to the specific intrachain chemistry - the intrachain LJ energy for the faster-cooled sample is even lower than for the slower cooled one, thereby making the total contribution of the LJ energy less significant. An explanation for the observed increase in intrachain LJ energy for the older sample will be given in $\S \mathrm{VA}$, when the stress is partitioned as well.

The interchain energy shown in fig. $7(\mathrm{~b})$ is displayed both in units of $\mathrm{J} \mathrm{g}^{-1}$ and in units of $\epsilon_{\mathrm{LJ}}$, where $\epsilon_{\mathrm{LJ}}$ equals the minimal energy of the LJ potential of all united atoms except the backbone $\mathrm{CH}$ united atoms (which is smaller by a fraction of 0.75 ). Here one can see that before de- 
formation a united atom in the slowly cooled sample has on average about $-3.85 \epsilon_{\mathrm{LJ}}$ interchain energy. Since this is smaller than the minimum of the LJ potential, each united atom should have on average multiple interchain bonds (i.e., a LJ-bond coordination number which is at least larger than 3). A large part could be due to phenylphenyl ring interactions, as each phenyl ring in the PS model consists of six united atoms. If two phenyl rings happen to be close and parallel, one united atom already has six close interchain interactions with other united atoms.

The difference between the slower and the faster-cooled sample is that the interchain energy per united-atom particle is on average approximately $0.1 \epsilon_{\mathrm{LJ}}$ lower for the slower-cooled sample prior to deformation. During deformation the difference does not vanish entirely; a plausible reason for this observation will be given in $§ \mathrm{IV} F$.

To yield the slowly cooled sample an increase in the interchain LJ energy is necessary: on average $0.2 \epsilon_{\mathrm{LJ}}$ per united atom. For the faster-cooled sample the binding energy is less, so also less bonds need to be broken to yield the material, suggesting that this is the reason that less force is needed to yield the younger material. The same effect could explain mechanical rejuvenation; if weak LJ bonds are already broken in the mechanical pretreatment by for example rolling ${ }^{49}$, then these bonds need not to be broken in the tension test and it would be likely that the resulting stress-strain curve would then show a much less pronounced or even absent yield tooth. In other polymers such as polycarbonate one would expect that there are less interchain LJ bonds to be broken to flow (as the backbone phenyl rings cannot come close to other chains) so that softening is expected to be smaller as well. Note that it takes time to reform and equilibrate the broken LJ bonds; as a consequence the total interchain LJ energy effectively becomes less negative during the initial straining region of net bond breaking.

\section{Connection with density}

As observed, more interchain LJ bonds are present in the more aged sample, which logically implies an increase in the yield-tooth stress. The evolution of the density during deformation (fig. 8) is supporting this view, as it is similar to the evolution of the interchain LJ energy (fig. 7(b)). Upon straining the density quickly decreases. This dilation is quantified by the Poisson ratio, the ratio of the strain in one of the perpendicular directions $\varepsilon_{\perp}$ to the strain in the extension direction $\varepsilon_{\|}$in the limit of infinitely small strain

$$
\nu=-\lim _{\varepsilon \rightarrow 0} \frac{\varepsilon_{\perp}}{\varepsilon_{\|}}
$$

As can be seen in table I our present and previous ${ }^{25}$ simulation results are in accordance with experimental values for polystyrene. Another way of interpreting the fast initial density decrease is that the Young modulus $E$ is

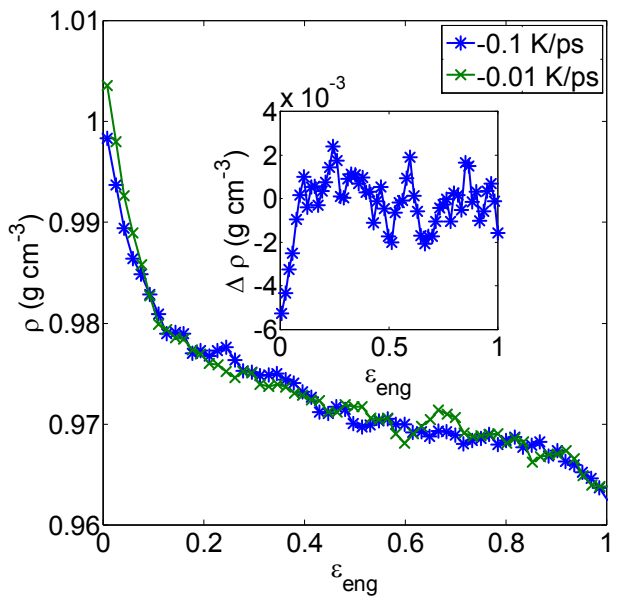

Figure 8: Density vs. strain for atactic PS for two different cooling rates during uniaxial-stress extension. Inset shows the difference. The initial difference in density disappears after yield.

not negligible with respect to the bulk modulus $K$, as $\nu=\frac{3 K-E}{6 K} 54$. This was also observed in a simulation of a PE-like model ${ }^{19}$. For larger strains $\left(\varepsilon_{\text {eng }}>0.1\right)$, the density decreases only weakly and the difference between the annealed and the quenched samples has disappeared. To sum up, the quenched and the annealed samples show next to a similar stress response also a similar density response for large strains.

\section{E. The relation with out-of-cage escape}

The breaking of LJ bonds under deformation, means that united atoms are forced to depart from their original cages. In our previous article ${ }^{70}$ we have shown that the cage in an undeformed glassy material such as PS manifests itself as a plateau in the root-mean-square translational displacement (RMSTD) of constitutive particles vs. time. After the plateau there is an increase in the RMSTD associated with cage escape. Deformation should therefore lead to an early increase in the RMSTD. Would the effect of thermal history or varying pressure also be visible in the RMSTD?

In fig. 9 the $\operatorname{RMSTD}\left\langle\Delta \mathbf{r}(t)^{2}\right\rangle^{1 / 2}=\left\langle\left(\mathbf{r}\left(t_{0}+t\right)-\right.\right.$ $\left.\left.\mathbf{r}\left(t_{0}\right)\right)^{2}\right\rangle^{1 / 2}$ averaged over all united atoms is plotted as a function of time $t$ both for the deformed and the undeformed case. For the deformed case the trivial convective velocity is removed as described in more detail by Lyulin et al. ${ }^{26}$ and in a future publication of ours. The resulting RMSTD will be also called the root-mean-square nonaffine displacement or simply non-affine displacement. In the case of deformation there is no translational invariance in time present and we therefore take $t_{0}$ as the time when the sample is unstrained, i.e., at $\varepsilon=0$. Later times $t_{0}+t$ will correspond to a strained sample at a strain value 
as given by the additional axis in fig. 9. The results are plotted for three situations: the annealed sample, the quenched sample and the sample under a high external pressure $\left(P_{\perp}=628 \mathrm{MPa}\right)$.

The RMSTD is associated with two basic phenomena: the temporary localization plateau and the cage-to-cage motion. The first focus is the localization plateau of the RMSTD. Its value is a measure for the space within the cage $^{70}$. If particles are closer to each other, we expect that the space within the cage is smaller. A higher density therefore implies a lower plateau value. Prior to deformation the fast-cooled sample has $\rho=1.001 \mathrm{~g} \mathrm{~cm}^{-3}$ (fig. 8), the slowly cooled sample $\rho=1.006 \mathrm{~g} \mathrm{~cm}^{-3}$ and the high-pressure sample $\rho=1.11 \mathrm{~g} \mathrm{~cm}^{-3}$. This order in density is consistent with the observed order in plateau values in fig. 9 .

Note that an aging effect is also visible in the highpressure plateau. When preparing the high-pressure sample a $0.5 \mathrm{~ns}$ equilibration at that pressure preceded the unstrained, isotropic production run (as mentioned in $\S I I)$. This equilibration time is even visible in the RMSTD plateau: while the two other samples (slowly and fast cooled) show a minor steady increase for the plateau value for larger time scales, the high-pressure sample shows a slight step near $0.5 \mathrm{~ns}$, reminiscent of the continuing aging.

The second point to observe in fig. 9 is the much earlier cage escape due to deformation (the cage escape time for the isotropic cage even falls out of the total simulation time). It is a signature of local mechanical rejuvenation as the local cage structure is destroyed. The cage escape looks quite similar for all three different situations. Only there seems to be a trend that the more bound cases (i.e., the cases with the lowest RMSTD plateau values) also have a smaller non-affine displacement after cage escape. This could be simply caused by the following. Assume that the position of a particle $\mathbf{r}\left(\varepsilon_{\mathrm{eng}}\right)$ at $\varepsilon_{\mathrm{eng}}=$ 0 can be written as $\mathbf{r}(0)=\mathbf{r}_{\min }(0)+\Delta \mathbf{r}_{\text {rat }}$, in which $\mathbf{r}_{\min }(0)$ is the quasi-equilibrium position of the particle in the unstrained glass, while rattling takes place in the direction $\Delta \mathbf{r}_{\text {rat }}$. Then

$$
\begin{aligned}
\left\langle\left(\mathbf{r}\left(\varepsilon_{\text {eng }}\right)-\mathbf{r}(0)\right)^{2}\right\rangle= & \left\langle\left(\mathbf{r}\left(\varepsilon_{\text {eng }}\right)-\mathbf{r}_{\min }(0)\right)^{2}\right\rangle+\left\langle\Delta \mathbf{r}_{\text {rat }}^{2}\right\rangle \\
& +\left\langle\left(\mathbf{r}\left(\varepsilon_{\text {eng }}\right)-\mathbf{r}_{\min }(0)\right)\left(\Delta \mathbf{r}_{\text {rat }}\right)\right\rangle .
\end{aligned}
$$

Assuming that the rattling motion is uncorrelated with $\mathbf{r}\left(\varepsilon_{\text {eng }}\right)-\mathbf{r}_{\min }(0)$, the last term in eq. 8 vanishes. Therefore the more bound states (i.e., with a lower $\left\langle\Delta \mathbf{r}_{\text {rat }}^{2}\right\rangle$ ) have a lower total $\operatorname{RMSTD}\left\langle\left(\mathbf{r}\left(\varepsilon_{\text {eng }}\right)-\mathbf{r}(0)\right)^{2}\right\rangle^{1 / 2}$, even when the particles do not only rattle, but diffuse as well.

\section{F. Mechanical erasure?}

Although some properties become independent of the thermal history after straining, differences still last for other ones. From our point of view this is to be expected. A small strain will obviously change the local

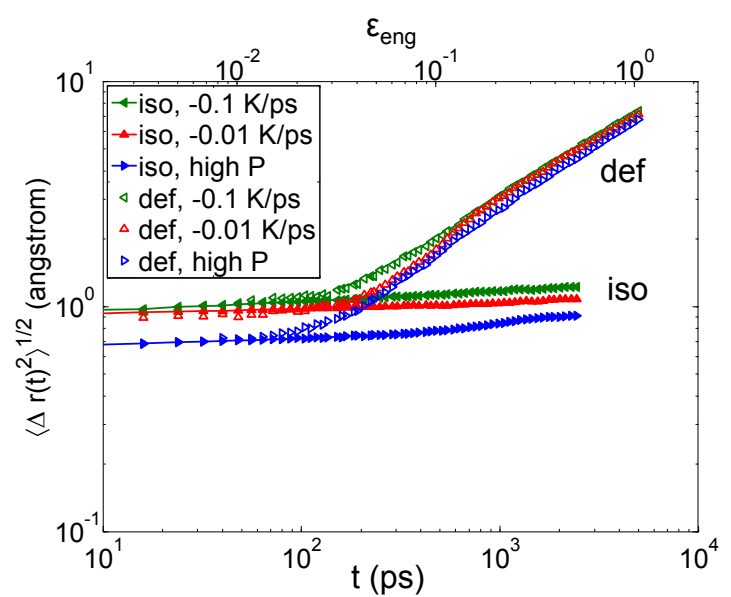

Figure 9: Root-mean-square displacement of united atoms for the slowly cooled, fast-cooled and high-pressure $(P=$ $628 \mathrm{MPa}$ ) samples in the undeformed ('iso', closed markers) and the deformed ('def', open markers) cases as a function of strain (for the deformed samples) and time.

structure. However, the erasing effect on much larger scales will be much less. An example of a measure of the large-scale structure in linear polymer chains is the characteristic ratio $C_{n}{ }^{44}$. If $n$ corresponds to the distance between the first and last segment of the chain, $C_{n}$ corresponds to the normalized end-to-end distance. For PS it is known that $C_{n}$ is temperature-dependent ${ }^{71}$. Also, both differently cooled samples fall out of equilibrium in a different way (such as at different cooling-rate dependent temperatures). As the end-to-end distance has a very long relaxation time and it is associated with larger lengthscales than the typical cage size, it will be out of equilibrium in the glassy state. Moreover, this out-of-equilibrium situation will be different for the two cooling scenarios. Therefore, the characteristic ratio for the two samples will differ as well. In particular the endto-end distance will be different and the difference will not be erased after initial yielding, because the end-toend distance of a long polymer chain will approximately be transformed affinely for small strains. We think that this reasoning even applies for experimental results, as in a glass phase the relaxation time of chain diffusion can easily exceed the duration of a typical experiment.

This temperature dependence of the chain structure can also explain the lasting difference in interchain energy between the two samples during deformation. The characteristic ratio for PS increases with lower temperatures $^{72}$. It is therefore likely that the characteristic ratio of the slower-cooled sample has a higher value - it had more time to adjust at a certain temperature. It turns out that this is indeed the case in our simulation results: for the 'older' chain $C_{50}=4.6 \pm 0.3$, while for the 'younger' chain $C_{50}=4.2 \pm 0.3$ for intrachain distances separated by 50 backbone bonds (for larger dis- 
tances the error in $C_{n}$ with respect to the difference in $C_{n}$ between the two cooling scenarios tends to increase). This means that the 'older' chain is more extended and is thus penetrating more in regions of overlap with other chains. Hence it is more likely that the annealed chain has more LJ interactions with other chains. This argument explains why the slowly cooled sample has a lower total interchain LJ energy, even after yielding, as was shown in fig. $7(\mathrm{~b})$.

Note that this is not in contradiction with the simulation of a binary LJ glass, in which the deformation did induce complete erasure ${ }^{73}$. In this simple glass the structure at scales larger than about two atom diameters looked identical in terms of the pair distribution function $g(r)$ for samples of different thermal history, so that in contrast to our polymer system no thermal-historydependent ordering was visible for large length scales.

The chain shape depends on its conformation, such as the trans and gauche probabilities, and these probabilities are temperature dependent. Moreover, the chain has a spectrum of length scales with accompanying relaxation times. Depending on the exact thermal history, each length scale can fall out of equilibrium at a different temperature. Therefore, many ordering parameters describing the non-equilibrium state of PS would be necessary (in the language of the Kovacs-Aklonis-HutchinsonRamos (KAHR) model ${ }^{74}$ ).

Our observed discrepancy from complete mechanical erasure of the thermal history is in line with other results. In the random-landscape model by Isner and Lacks ${ }^{75}$, in which the state of the material is given by a position in this energy landscape and strain is simply associated with a displacement in the energy landscape, the nonequilibrium state is also not only defined by one thermal and/or mechanical history-dependent variable and therefore also needs more ordering parameters. It seems thus that it has some long-range structure, too. Experimental studies of PS by means of positron-annihilation lifetime spectroscopy ${ }^{76}$ revealed as well that the complete erasure of thermal history by deformation is a too simplistic picture.

\section{STRESS PARTITIONING}

From the study of the changes in energetic contributions during uniaxial extension deformation it was observed that interchain interactions were most prominent near yield, while intrachain interactions dominate the strain-hardening regime. In this part we want to see if this is consistent with the partitioning of stress interactions. First we focus on the unstrained, isotropic situation $(\varepsilon=0)$. Although the total stress is simply connected to the pressure, the values of the various stress contributions are not. This analysis is followed by a discussion around the yield point for the deformed polymer. Finally, the stress partitioning in the strain-hardening regime is discussed. The stress-partitioning in this last

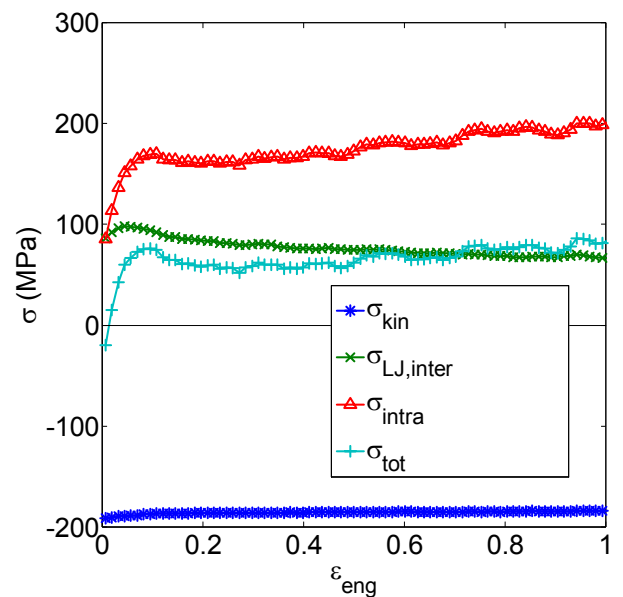

Figure 10: True stress in the extension direction vs. strain during uniaxial-stress extension, for different stress contributions. The hardening is mainly due to intrachain interactions. Interchain stress decreases after the yield peak, likely caused by the simultaneous decrease in density after yield. The yield tooth (peak and further softening) is caused by a combination of intrachain and interchain interactions.

regime gives more insight in the observed difference between compression and extension (fig. 1).

\section{A. Undeformed state}

We first concentrate on the undeformed case, $\varepsilon=0$. In fig. 10 the absolute true stresses along the uniaxial-stress extension direction are plotted as a function of strain. The total stress has been partitioned into several terms: the kinetic stress $\sigma_{\text {kin }}$, the intrachain stress $\sigma_{\text {intra }}$, and the interchain stress $\sigma_{\mathrm{LJ}, \text { inter }}$.

The kinetic stress is $\sigma_{\text {kin }}=-P_{\text {kin }}=-\rho_{N} k_{B} T \approx$ $-200 \mathrm{MPa}$ with $\rho_{N}$ the (united atom) number density. For the normal pressure simulations the imposed pressure is the offset pressure $P_{\perp}=42 \mathrm{MPa}$ (due to the density correction, §II), which is lower than $P_{\text {kin }}$. The negative kinetic stress term, naturally, is an expanding term to the total stress (i.e., due to the kinetic term particles repel each other). It is counterbalanced by the positive interchain and intrachain stress terms, which lead to contracting contributions. At $\varepsilon=0$ the intrachain stress and the interchain stress are of the same magnitude.

Upon comparing the LJ stress with previous literature, the closest work is that of a Monte Carlo simulation study of a polymeric network ${ }^{29}$. In that work a negative total LJ stress was found. There the total LJ stress was not split into a intrachain and interchain contribution. A similar result (i.e., a negative LJ stress) is present in our simulations. We can see this if the intrachain stress is partitioned further. This intrachain stress is composed of the stress due to covalent bonds $\sigma_{12}$, the stress due 
to the valence-angle interaction $\sigma_{13}$, the stress due to both the proper and improper-torsion interactions $\sigma_{14}$, and the stress due to intrachain LJ interactions $\sigma_{\mathrm{LJ}, \text { intra }}$ $\left(\sigma_{\text {intra }}=\sigma_{12}+\sigma_{13}+\sigma_{14}+\sigma_{\mathrm{LJ}, \text { intra }}\right)$. The $\sigma_{\mathrm{LJ}, \text { intra }}$ of PS turns out to be negative as well and the effect is even stronger than for the PE model: at $\varepsilon=0$ the stress $\sigma_{\text {LJ,intra }}$ is approximately equal to $-4 \mathrm{GPa}$, about one order of magnitude larger than for the PE-like network model $^{29}$.

The observation that the total LJ stress is negative can be understood by the following argument. Different from a linear united-atom PE chain, PS has side groups. In case of an all-trans configuration, the two neighbor phenyl rings of a meso dyad would be very close to each other (if assumed to be planar), well below the distance at which the LJ potential is at its minimum. The carbon atoms of these phenyl rings that are covalently bonded to the backbone are separated from each other by more than three chemical bonds (the minimum length at which LJ interactions play a role in case of intrachain interactions); hence they will exert in the currently adopted force field a very repulsive LJ interaction, making the associated $\sigma_{\mathrm{LJ}, \text { intra }}$ negative. As a reaction, the bonds in between will be extended, causing an attractive stress contribution (i.e., the bond wants to contract). From the simulation results it follows that there is indeed a big attractive intrachain stress contribution; also $\sigma_{12}$ has a very large value at $\varepsilon=0$, over $4 \mathrm{GPa}$. As expected, these high intrachain stress values almost cancel each other; the sum of $\sigma_{12}$ and $\sigma_{\mathrm{LJ}, \text { intra }}$ is less than $100 \mathrm{MPa}$. Therefore it is important to look at the total intrachain stress and not only at one of its constituents, as this gives rise to non-universal chemistry-specific effects.

\section{B. Yield regime}

Now let us take a closer look in what way these stress contributions in fig. 10 change upon straining the material. We concentrate first on the regime near the yield point. The yield peak is caused both by intrachain and interchain contributions. Each shows a yield tooth (peak with subsequent drop) in the stress, at around 5-10\% extension, although that of interchain nature occurs at a slightly smaller strain value. The dominant contribution to the total increase in the true stress from $\varepsilon=0$ till yield can be ascribed to the intrachain stress, in contrast to what was found for a melt of a freely-rotating chain, in which the interchain nonbonded-stress difference is the dominant contribution ${ }^{6}$. It is not clear what the reason is for this difference, although the force fields differ a lot from each other. Perhaps this illustrates that the current observation is of a non-universal nature and that the main resistance to initial flow can be either of interor intrachain nature.

It is instructive to also compare the stress partitioning of the two samples with different thermal histories, because we observed that the slower-cooled sample has a higher yield peak than the faster-cooled sample (fig. 2). Both intrachain and interchain stresses are responsible for this difference in yield peak, see fig. 11(a). We saw that only one energy increase is dominant near the yield peak (as found from the energy-partitioning results, $\S \mathrm{IV} B$ ). This is not in contradiction with each other. For the slower-cooled sample the interchain LJ interactions have a lower energy, i.e., a strong bond. To break this LJ bond a larger force is thus needed. If certain particles are dragged out of the LJ well, then neighboring covalently bonded particles will be moved along. Therefore, both the stress associated with the covalent bond and the stress associated with the LJ interaction need to be higher for the slower-cooled sample, in agreement with the present simulation results.

\section{Strain-hardening regime}

In the strain-hardening regime two facts are apparent from the stress partitioning (fig. 10). First, the intrachain interactions give rise to a positive contribution to the strain-hardening modulus. Second, the interchain interactions lead to a negative contribution. A probable cause of the latter is that there is a simultaneous decrease in density in this region (fig. 8), making it likely that interchain LJ bonds become weaker or are even broken. During extension the stress support of the interchain bonds obviously decreases or vanishes (in case they are broken). The value of $\sigma_{\text {kin }}$ also increases slightly due to the decrease in density upon the uniaxial-stress extension. Under compression, however, there is no decrease in density after yield. We indeed find that in this case the interchain stress does not decrease upon compression, see the results of partitioning the stress in case of a uniaxial-stress compression simulation in fig. 11(b).

The first observation, meaning that the positiveness of the strain-hardening modulus stems from intrachain interactions implies that the strain hardening is mainly carried by intrachain stresses. From one point of view this behavior seems logical, as the interchain bonds are broken during yielding (apart from entanglements), while the intrachain bonds are much stiffer and can withstand more stress before breaking (in our simulations the covalent bonds are even not allowed to break).

Can we also understand the observed difference in the strain-hardening modulus between the compression and the extension result for large strains (fig. 1)? The reason that we would expect similar moduli stems from assuming a simple strain-energy function $U$ of quadratic form $U\left(\lambda_{1}, \lambda_{2}, \lambda_{3}\right)=C\left(\lambda_{1}^{2}+\lambda_{2}^{2}+\lambda_{3}^{2}-3\right)$ (with $C$ a positive constant and $\lambda_{1}, \lambda_{2}$ and $\lambda_{3}$ the chain stretches along the three principal axes ${ }^{56}$ ), so that the state of minimal energy is the unstrained chain. If the material behaves incompressible, then the outcome of such a strain-energy function is that the strain-hardening modulus under compression is the same as under extension. In fact, the density is not constant but decreasing during extension 


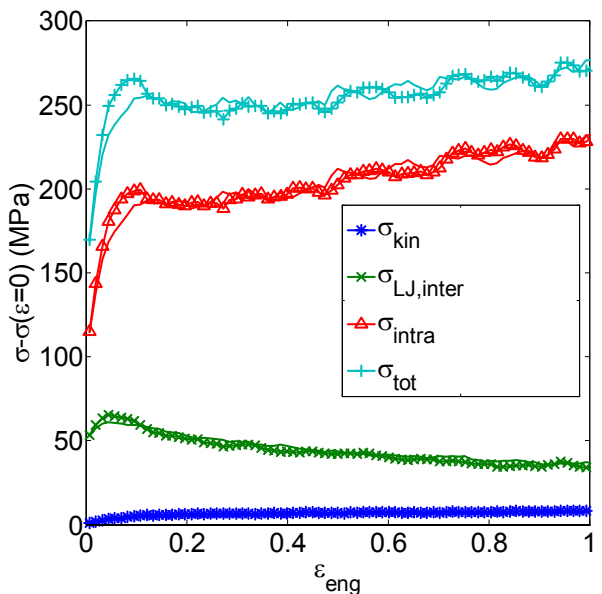

(a)

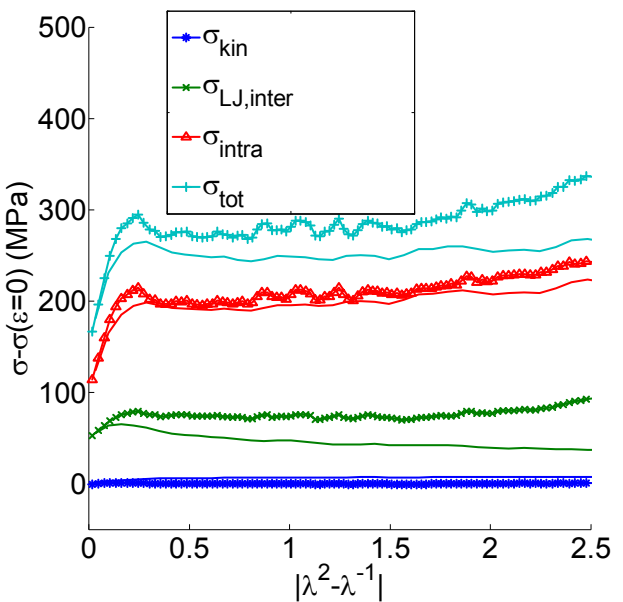

(b)

Figure 11: True stress in the active direction vs. strain for different stress contributions. The stress at $\varepsilon=0$ is subtracted and a separation of $50 \mathrm{MPa}$ between the different contributions is added for clarity. (a) Slowly (with markers, upper curve near yield) vs. the fast-cooled (without markers, lower curve near yield) sample. One can see that both the interchain and intrachain stress is higher for the slowly cooled sample. (b) Compression (with markers) vs. extension (without markers). The absolute Gaussian strain $\left|\lambda^{2}-\lambda^{-1}\right|$ is used for the $x$-axis and for compression the stresses have been multiplied by -1 to allow for a better comparison with extension. While for extension the interchain stress decreases after initial yielding, it is for compression fairly constant in the displayed range after initial yielding.

(fig. 8). This decrease in density is accompanied by a net breaking of interchain LJ bonds - the stress necessary to extend the sample further by breaking interchain LJ bonds therefore decreases. As concluded from the analysis of the yield peak both the interchain LJ stress and the intrachain stress are responsible for the breaking of interchain LJ bonds. So, although not yet perfect, we expect

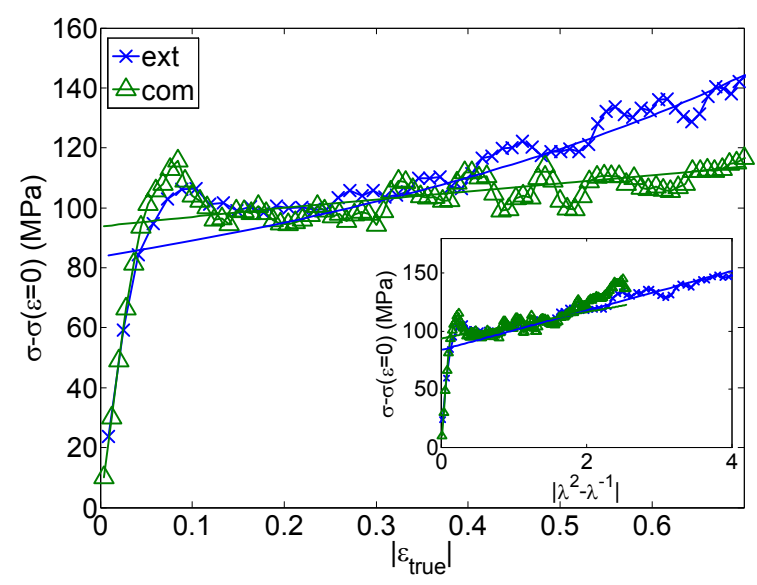

Figure 12: True intrachain stress in the active direction vs. absolute true strain for $(\triangle)$ compression and $(\times)$ extension. The stress at $\varepsilon=0$ is subtracted. For compression the stresses have been multiplied by -1 to allow for a better comparison with extension. Inset shows the same as a function of $\left|\lambda^{2}-\lambda^{-1}\right|$. Results of fitting the data by the Gaussian-based constitutive equation 2 are that $G_{h}=26 \mathrm{MPa}$ (compression) and $G_{h}=17 \mathrm{MPa}$ (extension).

that upon excluding the interchain LJ stress, the remaining stress behaves more as if the sample was incompressible. If we exclude the interchain LJ stress and only take into account the intrachain stress for determining $G_{h}$ by fitting eq. 2 , then the result is $G_{h}=26 \mathrm{MPa}$ for compression and $17 \mathrm{MPa}$ for extension (see also fig. 12). Note that these values are indeed much closer to each other than when the strain-hardening moduli are determined by fitting the total stress (compression: $G_{h}=37$, extension: $G_{h}=11 \mathrm{MPa}$, fig. 1). To sum up, the observed difference in strain hardening moduli between compression and extension is for a large part determined by the decrease in interchain stress for large strains under extension.

\section{SUMMARY AND CONCLUSIONS}

We have demonstrated that many mechanical properties of polystyrene can be simulated by MD in qualitative and sometimes even in quantitative agreement with experiments. From these simulations we conclude the following. The major difference between polystyrene chains of a different thermal history lies in the interchain LJ energy. A more annealed sample has more effective LJ bonds, i.e., the chains tend to stick more to each other during aging. These bonds should be broken in order to yield the material. Therefore, the yield stress is higher for the more aged sample. After some LJ bonds have been broken, the stress needed to deform decreases (strain softening). This breaking of bonds disturbs the thermal history at local scales and therefore both quenched 
and annealed samples soften to approximately the same yield minimum. However, differences in structure at large scales (due to the temperature-dependent chain structure) persist. Upon straining further the major contribution to final hardening is due to intrachain interactions. This conclusion is valid for uniaxial-stress compression as well as extension. We expect that, in contrast to PS, PC has fewer effective interchain LJ interactions which need to be broken during deformation, making the yield peak less pronounced and therefore leading to less brittle behavior.

We have shown that there is also an influence of pressure on the behavior at large strains: the strainhardening modulus increases with increasing pressure. The magnitude of this effect is in contradiction to the classical entropy-based rubber-elasticity picture and the inclusion of it can lead to better constitutive modeling. In the strain-hardening regime most of the applied work is dissipated. As the yield stress also increases with in- creasing external pressure, and the toughness of the material is characterized by the ratio of the strain-hardening modulus to the yield stress (according to Considère's construction), a higher external pressure does not lead to a significant increase in toughness. It would be very interesting to see if these new pressure results can be reproduced experimentally.

\section{Acknowledgment}

This work was partly financed by the Dutch Polymer Institute (DPI), project 487. This work was further supported by the Stichting Nationale Computerfaciliteiten (NCF), with funding of the Nederlandse Organisatie voor Wetenschappelijk Onderzoek (NWO). The Dutch nanotechnology network NanoNed is also acknowledged for their sponsorship.
1] R. N. Haward, Macromolecules 26, 5860 (1993).

[2] L. E. Govaert and T. A. Tervoort, J. Polym. Sci.: Polym. Phys. 42, 2041 (2004).

[3] L. E. Govaert, H. G. H. van Melick, and H. E. H. Meijer, Polymer 42, 1271 (2001).

[4] H. G. H. van Melick, L. E. Govaert, and H. E. H. Meijer, Polymer 44, 2493 (2003).

[5] E. J. Kramer, J. Polym. Sci.: Polym. Phys. 43, 3369 (2005).

[6] J. Gao and J. H. Weiner, J. Chem. Phys. 103, 1614 (1995).

[7] J. Gao and J. H. Weiner, J. Chem. Phys. 103, 1621 (1995).

[8] J. Gao and J. H. Weiner, Macromolecules 25, 1348 (1992).

[9] J. P. Gao and J. H. Weiner, Macromolecules 29, 6048 (1996).

[10] J. Rottler and M. O. Robbins, Phys. Rev. E 64, 051801 (2001).

[11] J. Rottler and M. O. Robbins, Phys. Rev. E 68, 011507 (2003).

[12] J. Rottler and M. O. Robbins, Comput. Phys. Commun. 169, 177 (2005).

[13] M. L. Wallace and B. Joos, Phys. Rev. Lett. 96, 025501 (2006).

[14] J. Gao and J. H. Weiner, J. Chem. Phys. 97, 8698 (1992).

[15] R. S. Hoy and M. O. Robbins, J. Polym. Sci.: Polym. Phys. 44, 3487 (2006).

[16] R. S. Hoy and M. O. Robbins, Phys. Rev. Lett. 99, 117801 (2007)

[17] R. S. Hoy and M. O. Robbins, Phys. Rev. E 77, 031801 (2008).

[18] J. H. R. Clarke and D. Brown, Mol. Sim. 3, 27 (1989).

[19] D. Brown and J. H. R. Clarke, Macromolecules 24, 2075 (1991).

[20] J. I. McKechnie, R. N. Haward, D. Brown, and J. H. R. Clarke, Macromolecules 26, 198 (1993).

[21] L. Yang, D. J. Srolovitz, and A. F. Yee, J. Chem. Phys. 107, 4396 (1997).
[22] F. M. Capaldi, M. C. Boyce, and G. C. Rutledge, Phys. Rev. Lett. 89, 175505 (2002).

[23] F. M. Capaldi, M. C. Boyce, and G. C. Rutledge, Polymer 45, 1391 (2004).

[24] S. Shenogin and R. Ozisik, Polymer 46, 4397 (2005).

[25] A. V. Lyulin, N. K. Balabaev, M. A. Mazo, and M. A. J. Michels, Macromolecules 37, 8785 (2004).

[26] A. V. Lyulin, B. Vorselaars, M. A. Mazo, N. K. Balabaev, and M. A. J. Michels, Europhys. Lett. 71, 618 (2005).

[27] A. V. Lyulin and M. A. J. Michels, Phys. Rev. Lett. 99, 085504 (2007).

[28] A. Fortunelli, C. Geloni, and A. Lazzeri, J. Chem. Phys. 121, 4941 (2004).

[29] C. Chui and M. C. Boyce, Macromolecules 32, 3795 (1999).

[30] A. S. Argon, P. H. Mott, and U. W. Suter, Phys. Status Solidi B 172, 193 (1992).

[31] A. S. Argon, V. V. Bulatov, P. H. Mott, and U. W. Suter, J. Rheol. 39, 377 (1995).

[32] S. S. Jang and W. H. Jo, Polymer 40, 919 (1999).

[33] M. Hutnik, A. S. Argon, and U. W. Suter, Macromolecules 26, 1097 (1993).

[34] C. F. Fan, T. Cagin, Z. M. Chen, and K. A. Smith, Macromolecules 27, 2383 (1994).

[35] C. F. Fan, Macromolecules 28, 5215 (1995).

[36] J. Li, T. Mulder, B. Vorselaars, A. V. Lyulin, and M. A. J. Michels, Macromolecules 39, 7774 (2006).

[37] T. Mulder, J. Li, A. V. Lyulin, and M. A. J. Michels, Macromol. Theor. Simul. 16, 348 (2007).

[38] B. Vorselaars, A. V. Lyulin, and M. A. J. Michels, Macromolecules 40, 6001 (2007).

[39] A. S. Lemak and N. K. Balabaev, J. Comput. Chem. 17, 1685 (1996).

[40] P. Zoller and D. J. Walsh, Standard pressure-volumetemperature data for polymers, Technomic Pub. Co., Lancaster, PA, 1995.

[41] Z. Pu, in Polymer data handbook, edited by J. E. Mark, Oxford University Press, Inc., Oxford, 1999.

[42] M. P. Allen and D. J. Tildesley, Computer simulation of 
liquids, Clarendon Press, Oxford, UK, 1987.

[43] A. T. Boothroyd, A. R. Rennie, and G. D. Wignall, J. Chem. Phys. 99, 9135 (1993).

[44] P. J. Flory, Statistical mechanics of chain molecules, Hanser Publishers, Munich, Germany, 1989.

[45] Q. Sun and R. Faller, Macromolecules 39, 812 (2006).

[46] L. J. Fetters, D. J. Lohse, D. Richter, T. A. Witten, and A. Zirkel, Macromolecules 27, 4639 (1994).

[47] L. J. Fetters, D. J. Lohse, S. T. Milner, and W. W. Graessley, Macromolecules 32, 6847 (1999).

[48] M. Doi and S. F. Edwards, The theory of polymer dynamics, Clarendon Press, New York, USA, 1986.

[49] H. G. H. van Melick, L. E. Govaert, and H. E. H. Meijer, Polymer 44, 3579 (2003).

[50] O. A. Hasan and M. C. Boyce, Polymer 34, 5085 (1993).

[51] M. Wendlandt, T. A. Tervoort, and U. W. Suter, Polymer 46, 11786 (2005).

[52] R. Quinson, J. Perez, M. Rink, and A. Pavan, J. Mater. Sci. 32, 1371 (1997).

[53] W. D. Callister, Jr., Materials science and engineering: an introduction, John Wiley \& Sons, Inc., New York, USA, fourth edition, 1997.

[54] D. W. van Krevelen, Properties of polymers - their correlation with chemical structure; their numerical estimation and prediction from additive group contributions, Elsevier Science B.V., Amsterdam, The Netherlands, third edition, 1997.

[55] J. Lemaitre and J.-L. Chaboche, Mechanics of solid materials, Cambridge University Press, Cambridge, 1990.

[56] I. M. Ward and J. Sweeney, An introduction to the mechanical properties of solid polymers, John Wiley \& Sons Ltd, West Sussex, England, second edition, 2004.

[57] J. J. Wu and C. P. Buckley, J. Polym. Sci.: Polym. Phys. 42, 2027 (2004).

[58] R. N. Haward, Polymer 35, 3858 (1994).

[59] R. N. Haward and R. J. Young, The physics of glassy polymers, Chapman \& Hall, London, second edition,
1997.

[60] R. N. Haward, Polymer 28, 1485 (1987).

[61] S. S. Sarva, S. Deschanel, M. C. Boyce, and W. N. Chen, Polymer 48, 2208 (2007).

[62] A. D. Mulliken and M. C. Boyce, Int. J. Solids Structures 43, 1331 (2006).

[63] L. R. G. Treloar, The physics of rubber elasticity, Clarendon Press; Oxford University Press, Oxford New York, 3rd edition, 2005.

[64] T. A. Tervoort and L. E. Govaert, J. Rheol. 44, 1263 (2000).

[65] A.-R. A. F. Ragab and S. E. A. Bayoumi, Engineering solid mechanics: fundamentals and applications, CRC Press, Boca Raton, Fl., 1999.

[66] L. D. Landau and E. M. Lifshitz, Theory of elasticity, Pergamon, Oxford, 3rd. English edition, 1986.

[67] R. N. Haward, Thermochim. Acta 247, 87 (1994).

[68] S. V. Shenogin, G. W. H. Hohne, and E. F. Oleinik, Thermochim. Acta 391, 13 (2002).

[69] D. J. Wales, Energy landscapes, Cambridge University Press, Cambridge, UK, 2003.

[70] B. Vorselaars, A. V. Lyulin, K. Karatasos, and M. A. J. Michels, Phys. Rev. E 75, 011504 (2007).

[71] R. F. Rapold and U. W. Suter, Macromol. Theor. Simul. 3, 1 (1994).

[72] D. Y. Yoon, P. R. Sundararajan, and P. J. Flory, Macromolecules 8, 776 (1975).

[73] M. Utz, P. G. Debenedetti, and F. H. Stillinger, Phys. Rev. Lett. 84, 1471 (2000).

[74] A. J. Kovacs, J. J. Aklonis, J. M. Hutchinson, and A. R. Ramos, J. Polym. Sci.: Polym. Phys. 17, 1097 (1979).

[75] B. A. Isner and D. J. Lacks, Phys. Rev. Lett. 96, 025506 (2006).

[76] D. Cangialosi, M. Wübbenhorst, H. Schut, A. van Veen, and S. J. Picken, J. Chem. Phys. 122, 064702 (2005). 